The original article on 'Still's Disease' reprinted from Med.-chir. Trans. (1897). 80, 47, by kind permission of the Honorary Editors, the Royal Society of Medicine. The Society also kindly provided photostat copies of the article and gave facilities for the reproduction of the illustrations.

\title{
ON A FORM OF CHRONIC JOINT DISEASE IN CHILDREN
}

\author{
BY \\ GEORGE F. STILL, M.A., M.D., M.R.C.P. \\ Medical Registrar and Pathologist to the Hospital for Sick Children, \\ Great Ormond Street, London \\ Communicated by Dr. Archibald E. Garrod \\ Received April 23-Read November 10, 1896
}

The occasional occurrence in children of a disease closely resembling the rheumatoid arthritis of adults has been recognized for several years. The identity of the disease seen in children with that in adults has never, so far as I am aware, been called in question.

The purpose of the present paper is to show that although the disease known as rheumatoid arthritis in adults does undoubtedly occur in children, the disease which has most commonly been called rheumatoid arthritis in children differs both in its clinical aspect and in its morbid anatomy from the rheumatoid arthritis of adults; it presents, in fact, such marked differences as to suggest that it has a distinct pathology.

The cases hitherto grouped together as rheumatoid arthritis in children include, therefore, more than one disease; and it will be shown that there are at least three distinct joint affections which have thus been included under the one head, rheumatoid arthritis.

The paper is based on a study of twenty-two cases, almost all of which have been in the Hospital for Sick Children, Great Ormond Street. Nineteen of these I have had under personal observation.

It will be necessary first to describe briefly the disease to which I have referred as the subject of this paper, and subsequently to point out the features of its clinical course and morbid anatomy, wherein it differs from the rheumatoid arthritis of adults.

The disease may be defined as a chronic progressive enlargement of joints. associated with general enlargement of glands and enlargement of spleen.

The onset is almost always before the second dentition; ten out of twelve cases began before the age of six years, and of these eight began within the first three years of life: the earliest was at fifteen months.

Girls are more commonly affected than boys: seven of the twelve cases were girls, five were boys.

The onset is usually insidious; the child, if old enough, complains of stiff- 
ness in one or more joints, which slowly become enlarged, and subsequently other joints become affected; but occasionally the onset is acute, with pyrexia and, it may be, with rigors.

I wish to lay some stress on the character of the enlargement of the joints. It feels and looks more like general thickening of the tissues round the joint than a bony enlargement, and is correspondingly smooth and fusiform, with none of the bony irregularity of the rheumatoid arthritis of adults.

The absence of osteophytic growth and of anything like bony lipping, even after years have elapsed since the onset, is striking.

There is, I believe, never any bony grating, although creaking, probably either of tendon or of cartilage, is frequently present. There is no redness or tenderness of the joints, except in very acute cases. The absence of pain is generally striking, but it may be present in slight degree, especially on movement. Limitation of movement, chiefly of extension, is almost always present; the child may be completely bedridden owing to more or less rigid flexion of joints.

The extensive deformities of the hands described by Charcot as occurring in the rheumatoid arthritis of adults ('Maladies des Vieillards,' 2nd edit., p. 201) are unknown to me in this disease. Those most common so far as I have seen are, flexion of the wrist with slight deviation of the hand to the ulnar side, and slight flexion of the proximal, combined sometimes with slight flexion of the distal, inter-phalangeal joint. Rarely there is slight hyperextension of the metacarpo-phalangeal or proximal interphalangeal joint. The fingers may deviate very slightly to the radial side, but more often the deviation is at the proximal inter-phalangeal joint, and may be to either side; indeed, both directions may be seen in one hand. Adduction of the thumb was marked in one case.

The joints earliest affected were usually the knees, wrists, and those of the cervical spine; the subsequent order of affection being ankles, elbows and fingers. The sterno-clavicular joint was affected in two out of twelve cases; the temporo-maxillary in three. The affection is symmetrical. There is no tendency to suppuration nor to bony ankylosis. The muscles which move the diseased joints show early and marked wasting, which contrasts often strongly with the good nutrition of the rest of the body.

The electrical reactions both to faradism and galvanism were brisk in three cases tested, but not otherwise altered.

Perhaps the most distinctive feature in these cases is the affection of the lymphatic glands. The enlargement is general, but affects primarily and chiefly those related to the joints affected. The glands are separate, rather hard than soft, not tender, and show no tendency to break down. They may become so large as to be visible, but more often do not become larger than a hazel-nut. The enlargement seems to bear a definite relation to the progress of the disease in the joints. Slight affection of the glands is found very soon after the first symptoms of the joint affection, and as the latter increases the glands become larger. If the joint affection subsides, the glands become smaller, increasing again in size if the joints become worse. 
The glands most affected are the supra-trochlear, those along the brachial artery, and those in the axilla, also those in Scarpa's triangle, and deep in the iliac fossa along the iliac artery, and those in the posterior triangle of the neck. In one case I thought that the popliteal glands were enlarged, and in two cases there was some evidence clinically of enlarged mediastinal glands. I have never been able to make out enlargement of mesenteric glands, but in one of the autopsies which I shall mention, the glands in the hilum of the liver were found enlarged.

It will thus be seen that the enlargement is general; and I may add that it is constant; it was found in all the twelve cases mentioned.

Enlargement of the spleen is also a striking feature of these cases. It is, of course, not always easy to be certain of splenic enlargement, but it was definite and considerable in nine out of the twelve cases, the edge of the spleen being felt one to two fingers' breadth below the costal margin. The enlargement of the spleen seems to be roughly proportionate to that of the glands, and like that of the glands has been observed to inorease as the joint condition became worse.

The heart shows no evidence of valvular disease, but haemic bruits were detected in some of the cases.

There were some physical signs suggestive of adherent pericardium in two of the twelve cases, and in three other cases adherent pericardium was found quite unexpectedly at the autopsy.

Anaemia is generally present to some extent, but is seldom profound; the face has often a curious waxy pallor with flushed cheeks. The blood shows only moderate diminution of red corpuscles in most cases; in some, however, there is disproportionate deficiency of haemoglobin.

A curious symptom noticed in four cases out of the twelve, was slight prominence of the eyes, hardly enough to call exophthalmos, but enough to be noticeable. The thyroid seemed normal in the cases which I examined.

Sweating is often profuse, and not related to temperature. The temperature seems to be of two varieties: the one shows periods, generally lasting only a few days, of pyrexia followed by a longer interval of apyrexia; the other shows more or less continuous slight pyrexia. The pyrexial attacks occasionally show a curious regularity in their recurrence. One or two cases showed sudden attacks of hyperpyrexia, lasting one hour or two, and then subsiding rapidly. The pyrexial periods are not usually associated with any clinically demonstrable exacerbation of the joint trouble, nor indeed is it possible usually to find any definite cause for the fever.

I have made a detailed study of the urine in these cases, and find that the urea, uric acid, phosphoric acid and chlorides each show considerable variations, but that these variations are within the limits of health, as shown by a series of analyses of the urine from healthy children. The urine in other respeets also was normal.

A remarkable feature in these cases is the general arrest of development that occurs when the disease begins before the second dentition. A child of twelve-and-a-half years would easily have been mistaken for six or seven 
years, while another of four years looked more like two-and-a-half or three years.

The arrest is, however, of bodily rather than of mental development, and hence although backward in some respects from the enforced absence from school, the child often appears by comparison with its size to be rather precocious than backward.

The course of these cases is slow. Improvement may occur for a time under treatment or spontaneously, but the disease soon progresses again until a condition of general joint disease is reached which seems to be permanently stationary. The disease is not in itself fatal; the few deaths that have been recorded were due to complications.

Curiously enough, some accidental complications have been followed by marked improvement; thus I have known measles, scarlet fever and catarrhal jaundice, to be each followed by distinct improvement of the joint symptoms.

The etiology of the disease is very uncertain. A careful investigation of the family history in ten cases gave the following figures: phthisis in five, acute rheumatism in four, rheumatoid arthritis in one (a grandmother), gout in none, syphilis in none. The frequency of acute rheumatism and phthisis here cannot be considered to have any special significance; a similar investigation of fifty consecutive non-rheumatic, and also of fifty non-tubercular cases in the Hospital for Sick Children gave respectively 44 per cent. and 46 per cent. as the number of times that acute rheumatism and phthisis occurred in the family history.

No relation to diet in infancy could be shown. Poverty and exposure to insanitary conditions seemed to play little if any part in its causation.

The morbid anatomy of this disease is gathered from three post-mortems. (I unfortunately did not see these myself, so that my information is obtained from the post-mortem records, corroborated by those who saw the autopsies.) For permission to make use of the record of one of these cases I am indebted to the kindness of your President, Dr. Dickinson.

The joints show marked thickening of the capsule and of the connective tissue just outside this. There is also thickening and vascularization of the synovial membrane, and fibrous adhesions are sometimes present.

The cartilage may be perfectly normal, as in two cases that had lasted nearly one-and-a-half years; but in a case that had lasted three years it showed pitting of its surface as if from pressure, with little processes of the thickened synovial membrane fitting accurately into the pits, which were situated chiefly at the margin of the cartilage; otherwise, however, the cartilage was healthy-there was no fibrillation, no osteophytic change, no exposure or eburnation of bone.

The enlarged glands appear normal on section, or show small ecchymoses in their substance.

The spleen weighed in each case about $5 \mathrm{oz}$., so that it was considerably enlarged; it was firm, and appeared normal on section.

In each case the pericardium was universally adherent; there were also pleural adhesions. There was no endocarditis certainly in two cases, but in the third the mitral valve was perhaps a little thickened.

The following case, under the care of Dr. Lees at the Hospital for Sick 
Children, Great Ormond Street, may serve to illustrate some of the points mentioned.

Alice C., at the age of two years and four months began to limp in walking, and it was noticed that the ankles were swollen. A week or two later the elbows, and then the knees, became stiff and swollen; stiffness of the neck also was noticed almost from the first onset. The joints affected became steadily worse, and the child quickly lost the power of standing.

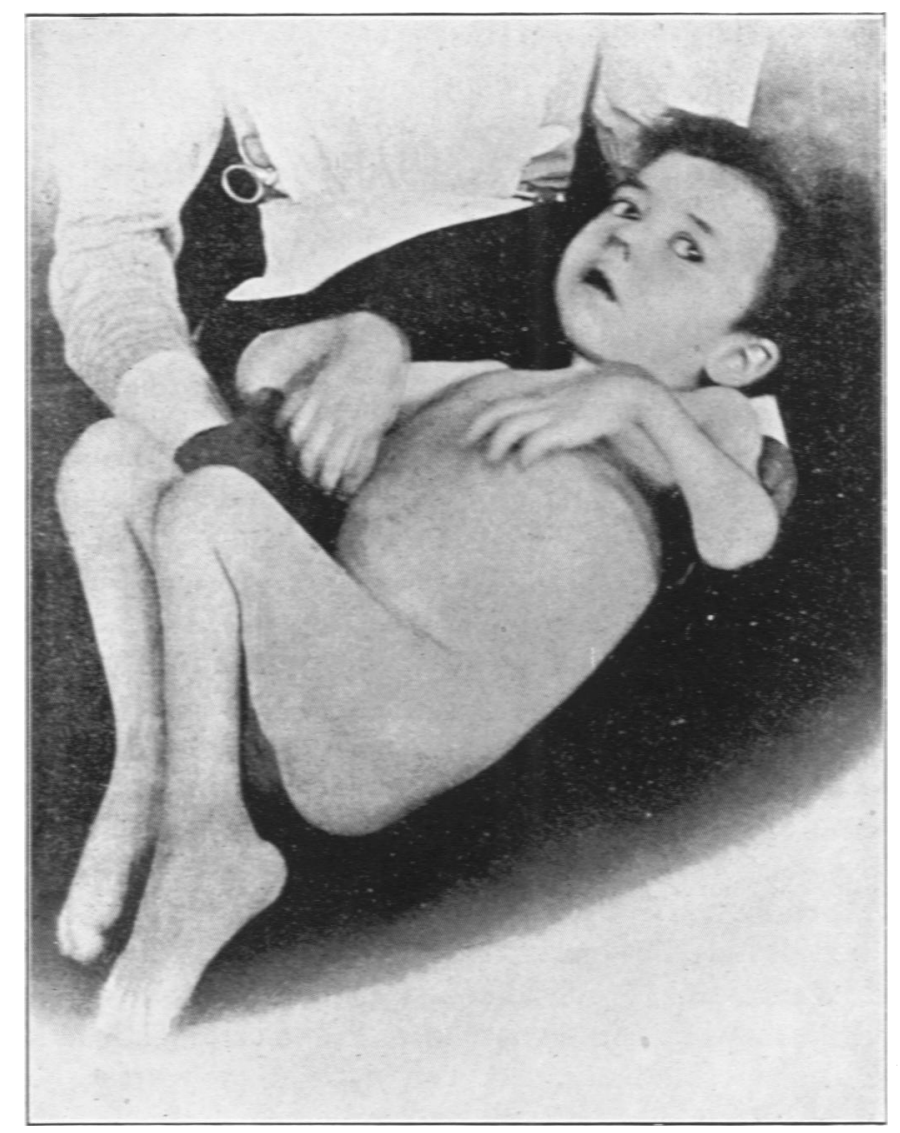

Chronic arthritis, with enlargement of glands and spleen.

Fig. 1.-Alice C., aged 4 years; showing tendency to fixation of joints in position of flexion.

There had been no previous illness, except whooping-cough; the child had been carefully hand-fed after it was four weeks old; up to this time it had been breast-fed. There had been no privations, but the house was damp. The family history showed nothing of importance except a doubtful history of rheumatism in two maternal uncles.

When first seen at the age of three-and-a-half years, the child was fairly nourished, with round face and slightly prominent eyes. All the joints of the limbs were affected; extension of the knees, hips and elbows was considerably limited, there was thought to be a little fluid in the knees, ankles and wrists, 
and there was obvious fusiform elastic thickening of all these joints except the shoulders and hips. I could not find any definite bony enlargement, and there was no grating. The sterno-clavicular and temporo-maxillary joints, and dorsal and lumbar spine, were unaffected.

Gland enlargement was marked; the axillary, supra-condylar, cervical, iliac and inguinal were affected; the axillary were easily visible. The glands were hard, separate, and not tender.

The spleen was one finger's breadth below the costal margin. Heart and lungs were normal.

For nearly two years the child has been in the Hospital for Sick Children, and the joint condition has slowly become worse (as will be seen from the photograph shown), so that the child is unable even to turn herself in bed. The progress of the disease has, however, not been steady; at one time there was a slight improvement in the joint condition, and synchronously with this the glands and spleen became much smaller. above.

The temperature chart has shown the recurrent attacks of pyrexia described

Having sketched the clinical aspect and morbid anatomy of the disease, I wish now to draw attention to the points in which the condition differs from the rheumatoid arthritis of adults.

The clinical features of the joint affection cannot be considered distinctive, but it may be pointed out that the fusiform enlargement which feels like extraarticular thickening of soft tissues, and the absence of bony lipping and grating, even after the disease has lasted some years, are very unlike the irregular bony enlargement of joints found in the advanced rheumatoid arthritis of adults.

Pathologically, however, the joints show marked differences. In the children's disease there is complete absence, even in an advanced case, of the cartilage changes which are seen quite early in the rheumatoid arthritis of adults. In the children's disease, also, there is a very considerable thickening of the capsule, and of the connective tissue just outside this, which is a much less prominent feature in the disease of adults.

On the enlargement of glands I lay great stress. It is, I think, one of the most important points of distinction clinically between this disease and rheumatoid arthritis. It is, as far as I know, never found in the rheumatoid arthritis of adults, whereas it is a constant symptom in the disease of children here described.

The enlargement of the spleen, associated with the glandular enlargement, is another important distinction, and like the preceding symptom is, I believe, unknown in adults.

Other minor differences are the following:

The incidence on the sexes is different. The proportion of females to males affected by rheumatoid arthritis in adult life was found by Sir A. Garrod to be $5: 1$, whereas in the disease described above the proportion is barely $1.5: 1$; my numbers, however, are so small that no great weight can be attached to this difference.

The order of affection of joints is different. In adults rheumatoid arthritis affects the small joints of the hands quite early, and often begins here; whereas the disease of children begins nearly always in the knees or wrists, and the fingers 
remain often free for months or even years. The very early and almost constant occurrence of affection of the cervical spine is also, I fancy, far more common in the disease here described.

Lastly, the occurrence of adherent pericardium, certainly in three, probably in five cases out of twelve, with no clinical evidence of endocarditis in any case, and only a slight thickening of the mitral valve of very doubtful significance found post-mortem in one case, and also the occurrence of pleurisy in four out of twelve cases, suggests some peculiar liability of children with this disease to inflammation of serous membranes, a liability which is not shared by the rheumatoid arthritis of adults.

From the foregoing it is seen that there are well-marked clinical and pathological differences between the rheumatoid arthritis of adults and the disease of children above described. But when it is suggested that the two conditions are therefore different diseases, it may be objected that the points of distinction mentioned may all be due merely to the difference of age.

This objection seems to me sufficiently answered by the fact that there occurs in children, rarely indeed, but only rather more rarely than the disease here described, a condition which appears to be identical in every respect with the rheumatoid arthritis of adults.

I shall now deal briefly with the second point, namely, that the cases hitherto grouped together as rheumatoid arthritis in children include at least three distinct conditions.

The least rare is the disease already described, which for the reasons I have given should not be called rheumatoid arthritis.

Next in frequency is a joint affection which cannot be distinguished from the rheumatoid arthritis of adults. It is unnecessary to describe it. It presents the same general enlargement of joints, with subsequent bony thickening and lipping, and bony grating as in adults. There is no enlargement of lymphatic glands or spleen, and no evidence of pericarditis.

Of the twenty-two cases, six were of this nature, and the age at onset was generally rather higher than in the preceding disease: two began before the second dentition, one at one year and eight months.

(In the photograph shown by kind permission of Dr. Cheadle. the contrast is seen between the fusiform enlargement of the phalangeal joints in the first disease, and the bony enlargement of rheumatoid arthritis.)

The third condition that has, I venture to think, been confused with rheumatoid arthritis is a form of rheumatism. It is extremely rare. I have seen only one case. It was in a boy, aged five years, under the care of Dr. Lees at the Hospital for Sick Children.

At the age of three-and-a-half years the fingers became stiff, apparently from the formation of fibrous nodules over the tendons. Then the elbows and wrists slowly enlarged, and their movements became limited. There was also some stiffness of the neck. There was no tenderness of joints, and no acute illness at any time. Seen one-and-a-half years later, the elbows and wrists showed firm thickening, very suggestive of extra-articular fibrous thickening. There was none of the fusiform elastic enlargement of the phalangeal joints 
which was seen in all the advanced cases of the disease described in the earlier part of this paper; indeed, there was no evident affection of the phalangeal joints, although there was marked limitation of movement, the terminal phalanges being flexed, and some of the proximal phalanges hyper-extended.

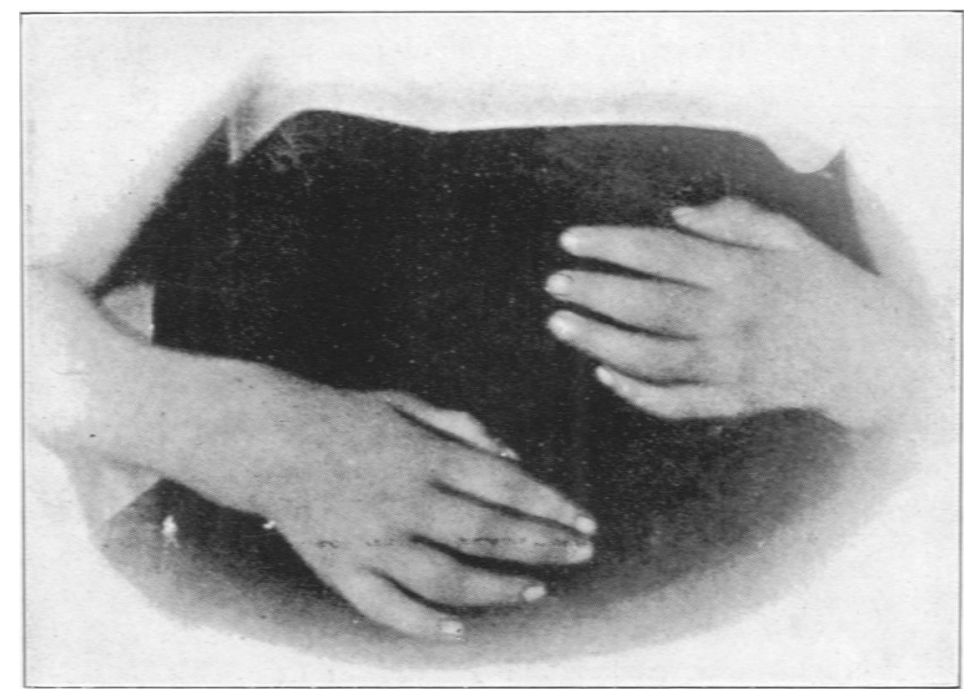

FIG. 2.-Hands of girl aged 7 years, showing fusiform enlargement of joints in the form of chronic arthritis described, with enlargement of glands and spleen.

There was a loud apical systolic bruit; there was no enlargement of spleen or glands. Subcutaneous fibrous nodules were present on the fingers, elbows and head.

In this particular case, although there was no history of acute rheumatism, the association of a cardiac bruit with fibrous nodules was, I think, sufficient proof of the rheumatic nature of the joint affection; but in cases where such additional symptoms were not present to aid the diagnosis, the disease might

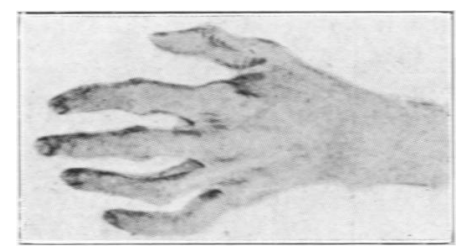

FIG. 3.- Hand of boy aged 10 years, showing bony thickening of joints in rheumatoid arthritis.

easily be mistaken for an early rheumatoid arthritis. There can, I think, be no doubt that it is identical with the disease described by Jaccoud (art. 'Polyarthrite Déformante,' Pathologie Interne, 1871) as chronic fibrous rheumatism.

It is particularly interesting to note that although Jaccoud described the condition as a disease of young adults, and occurring after repeated attacks of 
rheumatic fever, in the case I have mentioned the disease began at three-and-ahalf years of age, and there was never any acute rheumatism.

The points of difference between this and the preceding conditions are the following: The evidence of rheumatism, and this is the most diagnostic feature when present, distinguishes it from both the previously described diseases. The absence of bony thickening and of bony grating, and the character of the
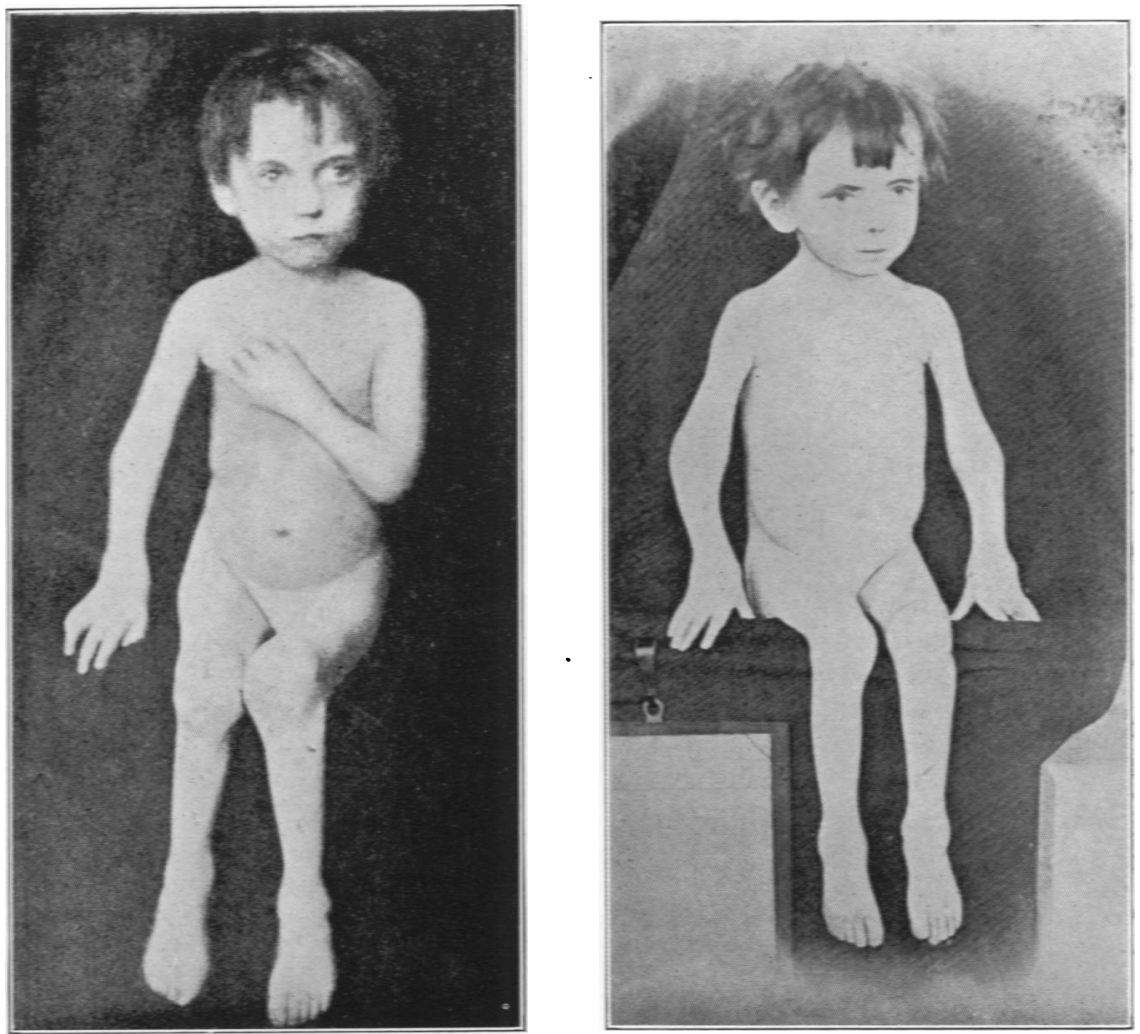

Chronic arthritis, with enlargement of glands and spleen.

FIG. 4.-Girl aged $3 \frac{1}{z}$ years; disease began at $2 \frac{1}{2}$ years.

Fig. 5.-Jane R., aged 4 years: disease began at the age of 15 months.

joint enlargement, which strongly suggests extra-articular fibrous thickening, and I think also the much less likelihood of affection of the small joints of fingers and toes, distinguish this disease from rheumatoid arthritis in children; while the absence of gland and spleen enlargement, and probably here also the less likelihood of affection of the small joints, distinguish it from the disease described in the first part of this paper.

In conclusion, I should like just to mention another affection which I have known raise the question of rheumatoid arthritis in a child. It is a rare form of syphilitic joint disease. It shows, in addition to the commoner chronic effusion with thickening of capsule in medium-sized and larger joints, definite bony thickening and lipping, which affects also the smaller joints; this osteo- 
phytic change may even simulate Heberden's nodes, as I have seen in a boy six years old, in whom some of the distal phalanges showed lateral thickenings very like Heberden's nodes. They were, however, less regular in their distribution, for they occurred not only on the distal, but also on one or two of the proximal phalangeal joints, and the toes showed similar nodosities. In this boy the larger joints showed chronic effusion, with some thickening of surrounding soft tissues, and there was a gumma over one ulna, and further evidence of syphilis was found in old iritis. There was no enlargement of glands or spleen.

It may be useful to sum up the conclusions arrived at in this paper.

There is a disease, occurring in children, and beginning before the second dentition, which is characterized clinically by elastic fusiform enlargement of joints without bony change, and also by enlargement of glands and spleen.

This disease has hitherto been called rheumatoid arthritis, but it differs from that disease in adults, clinically in the absence of bony change, even when the disease is advanced, and in the enlargement of glands and spleen, and pathologically in the absence, even in an advanced case, of the cartilage changes which are found quite early in that disease, and also in the absence of osteophytic change.

These differences are not to be attributed merely to modification of disease by difference of age, as there occurs also in children a disease in every respect identical with the rheumatoid arthritis of adults.

Under the head of rheumatoid arthritis in children, at least three conditions have been confused which are both clinically and pathologically distinct, namely: (1) The joint disease described in the present paper; (2) a disease identical with the rheumatoid arthritis of adults; (3) a disease probably identical with that described by Jaccoud as chronic fibrous rheumatism.

Before ending this paper, I wish to express my gratitude to Dr. Barlow, Dr. Lees, Dr. Penrose and Dr. A. E. Garrod, not only for kind permission to use their cases, but also for allowing me to bring forward this subject. They have, I know, taken great interest in these cases for several years, especially in the disease mentioned in the earlier part of this paper, and therefore several of the points which I have described had been already observed by them. I have to thank them for drawing my attention to some of these points, and for allowing me to make use of their observations. To Dr. Garrod I am indebted also for many valuable criticisms and suggestions.

Since the above paper was written, two French observers, Chauffard and Ramond (Revue de Méd., May, 1896), have described occasional enlargement of the lymphatic glands in adults with an acute form of rheumatoid arthritis, which they distinguish as 'infective arthritis'; similar cases have been observed by Drs. Bannatyne and Wohlmann of Bath (Lancet, April, 1896). The disease, however, which I have described seems to differ in several points from the conditions described by these observers. 